\title{
GENERALIZED PERIODIC AND GENERALIZED BOOLEAN RINGS
}

\author{
HOWARD E. BELL and ADIL YAQUB
}

(Received 21 August 2000)

\begin{abstract}
We prove that a generalized periodic, as well as a generalized Boolean, ring is either commutative or periodic. We also prove that a generalized Boolean ring with central idempotents must be nil or commutative. We further consider conditions which imply the commutativity of a generalized periodic, or a generalized Boolean, ring.
\end{abstract}

2000 Mathematics Subject Classification. 16D70, 16 U80.

Throughout, $R$ denotes a ring, $N$ the set of nilpotents, $C$ the center, and $E$ the set of idempotents of $R$. A ring $R$ is called periodic if for every $x$ in $R$, there exist distinct positive integers $m, n$ such that $x^{m}=x^{n}$. We now formally state the definitions of a generalized periodic ring and a generalized Boolean ring.

DEFINITION 1. A ring $R$ is called generalized periodic if for every $x$ in $R$ such that $x \notin(N \cup C)$, we have $x^{n}-x^{m} \in(N \cap C)$, for some positive integers $m, n$ of opposite parity.

DEFINITION 2. A ring $R$ is called generalized Boolean if for every $x$ in $R$ such that $x \notin(N \cup C)$, there exists an even positive integer $n$ such that $x-x^{n} \in(N \cap C)$.

THEOREM 3. If $R$ is a generalized periodic ring, then $R$ is either commutative or periodic.

Proof. Let $N$ and $C$ denote the set of nilpotents and the center of $R$, respectively. We distinguish three cases.

CASE $1(N \subseteq C)$. Then $x \notin C$ implies $x \notin(N \cup C)$, and hence there exist distinct positive integers $m, n$ such that $x^{m}-x^{n} \in N$, with $n>m$. Suppose $\left(x^{m}-x^{n}\right)^{k}=0$. Then, as is readily verified,

$$
\left(x-x^{n-m+1}\right)^{k} x^{k(m-1)}=0,
$$

which, in turn, implies that

$$
\begin{aligned}
\left(x-x^{n-m+1}\right)^{k m} & =\left(x-x^{n-m+1}\right)^{k} x^{k(m-1)} g(x) \\
& =0,
\end{aligned}
$$

where

$$
g(\lambda) \in \mathbb{Z}[\lambda] .
$$

We have thus shown that

$$
x-x^{n-m+1} \in N, \quad \forall x \notin C,(n-m+1>1) .
$$


Recall that, in our present case, we assumed that $N \subseteq C$, and hence by (4),

$$
x-x^{n-m+1} \in C, \quad \forall x \notin C,(n-m+1>1) .
$$

Since (5) is trivially satisfied if $x \in C$, we see that

$$
x-x^{n(x)} \in C, \text { for some } n(x)>1 \text {, where } x \in R \text { (arbitrary). }
$$

Therefore, $R$ is commutative, by a well-known theorem of Herstein [3].

CASE $2(C \subseteq N)$. Then $x \notin N$ implies $x \notin(N \cup C)$, and hence there exist distinct positive integers $m, n$ such that $x^{n}-x^{m} \in N$, with $n>m$. Repeating the argument used to prove (4), we see that

$$
x-x^{n-m+1} \in N, \quad \forall x \notin N,(n-m+1>1) .
$$

Since (7) is trivially satisfied for all $x \in N$, we conclude that

$$
x-x^{k(x)} \in N, \text { for some } k(x)>1 \text {, where } x \in R \text { (arbitrary). }
$$

By a well-known theorem of Chacron [2], equation (8) implies that $R$ is periodic.

CASE $3(C \nsubseteq N$ and $N \nsubseteq C)$. In this case, let

$$
z \in C \backslash N, \quad u \in N \backslash C .
$$

Equation (9) readily implies that $z+u \notin C$ and $z+u \notin N$, and hence (see Definition 1)

$$
(z+u)^{n}-(z+u)^{m} \in N, \text { for some integers } n>m \geq 1 .
$$

Since $z$ commutes with the nilpotent element $u$, (10) implies that

$$
z^{n}-z^{m}+u^{\prime} \in N, \quad \text { where } u^{\prime} \in N, u^{\prime} \text { commutes with } z \text {. }
$$

Hence $z^{n}-z^{m} \in N$, with $n>m \geq 1$. Now, a repetition of the argument used in the proof of (4) shows that

$$
z-z^{n-m+1} \in N, \quad \forall z \in C \backslash N,(n-m+1>1) .
$$

Trivially,

$$
x-x^{k} \in N, \quad \forall x \in N, \forall k \in \mathbb{Z}^{+} .
$$

Finally, if $x \notin(N \cup C)$, then

$$
x^{n}-x^{m} \in N, \text { for some integers } n>m \geq 1 .
$$

Again, repeating the argument used in the proof of (4), we see that

$$
x-x^{n-m+1} \in N, \quad \forall x \notin(N \cup C),(n-m+1>1) .
$$

Combining (12), (13), and (15), we conclude that

$$
x-x^{k(x)} \in N, \text { for some } k(x)>1 \text {, where } x \in R \text { (arbitrary). }
$$

Thus, by Chacron's theorem [2], $R$ is periodic. This completes the proof. 
COROLLARY 4. If $R$ is a generalized Boolean ring, then $R$ is either commutative or periodic.

This follows at once, since a generalized Boolean ring is necessarily a generalized periodic ring (see Definitions 1 and 2).

Before proving the next theorem, we prove the following lemma.

LEMMA 5. Let $R$ be a generalized periodic ring. If e is any nonzero central idempotent in $R$ and $a \in N$, then $e a \in C$.

Proof. The proof is by contradiction. Suppose the lemma is false, and let

$$
\eta_{0} \in N, \quad e \eta_{0} \notin C .
$$

Since $e \in C$ and $\eta_{0} \in N$, therefore $e \eta_{0}$ is nilpotent. Let

$$
\left(e \eta_{0}\right)^{\alpha} \in C, \quad \forall \alpha \geq \alpha_{0}, \alpha_{0} \text { minimal. }
$$

Since $e \eta_{0} \notin C$ (see $\left.(17)\right)$, therefore $\alpha_{0}>1$. Let $\eta=\left(e \eta_{0}\right)^{\alpha_{0}-1}$. Then,

$$
\begin{gathered}
\left.\eta=\left(e \eta_{0}\right)^{\alpha_{0}-1} \in N, \quad \eta \notin C \text { (by the minimality of } \alpha_{0}\right), \\
\eta^{k} \in C, \quad \forall k \geq 2, \quad e \in C, \quad e^{2}=e \neq 0, \quad e \notin N .
\end{gathered}
$$

Equation (19) implies that $e+\eta \notin C$ and $e+\eta \notin N$, and hence (see Definition 1)

$$
(e+\eta)^{m^{\prime}}-(e+\eta)^{n^{\prime}} \in C
$$

where $m^{\prime}, n^{\prime}$ are of opposite parity. Combining (20) and (19), we see that (keep in mind that $e \eta=\eta$; see (19))

$$
\left(m^{\prime}-n^{\prime}\right) e \eta \in C,
$$

where $m^{\prime}-n^{\prime}$ is an odd integer. Equation (19) also implies that $(-e+\eta)$ is not in $(N \cup C)$, so

$$
(-e+\eta)^{m^{\prime \prime}}-(-e+\eta)^{n^{\prime \prime}} \in N,
$$

where $m^{\prime \prime}, n^{\prime \prime}$ are of opposite parity. Combining (19) and (22), we see that

$$
(-e)^{m^{\prime \prime}}-(-e)^{n^{\prime \prime}} \in N
$$

and hence $2 e \in N$, since $m^{\prime \prime}$ and $n^{\prime \prime}$ are of opposite parity. Therefore, $(2 e)^{\gamma}=0, \gamma \in \mathbb{Z}^{+}$, and thus $2^{\gamma} e=0$, which implies that

$$
2^{\gamma} \text { e } \in C ; \quad \gamma \in \mathbb{Z}^{+} .
$$

Now, combining (21) and (24), keeping in mind that $\left(2^{\gamma}, m^{\prime}-n^{\prime}\right)=1$, we see that $e \eta \in C$, and hence, by (19), $\eta=e \eta \in C$, which contradicts (19). This contradiction proves the lemma.

As usual, $[x, y]=x y-y x$ denotes the commutator of $x$ and $y$.

We are now in a position to prove the following theorem. 
THEOREM 6. Suppose $R$ is a generalized periodic ring, and suppose that there exists an element $c$ in $C$, with $c \neq 0$, such that

$$
c[x, y]=0 \quad \text { implies }[x, y]=0, \forall x, y \in R \text {. }
$$

Then $R$ is commutative.

Proof. We distinguish two cases.

CASE $1(c \in N)$. In this case, $c^{k}=0$ for some positive integer $k$, and hence

$$
c^{k}[x, y]=0, \quad \forall x, y \in R
$$

Combining (25) and (26), we see that

$$
\begin{aligned}
c^{k}[x, y]=0 & \Longrightarrow c\left[c^{k-1} x, y\right]=0 \Longrightarrow\left[c^{k-1} x, y\right]=0 \Longrightarrow c^{k-1}[x, y]=0 \\
& \Longrightarrow \cdots \Longrightarrow c[x, y]=0 \Longrightarrow[x, y]=0 .
\end{aligned}
$$

Thus, $c^{k}[x, y]=0$ implies $[x, y]=0$, and hence $R$ is commutative.

CASE $2(c \notin N)$. In view of Theorem 3, we may assume that $R$ is periodic. This implies, in particular, that $c^{m}$ is idempotent for some positive integer $m$. Furthermore, $c^{m} \neq 0$ (since $c \notin N$ in our present case). The net result is (since $c \in C$ also)

$$
c^{m}=e \quad \text { is a nonzero central idempotent in } R \text {. }
$$

Let $a \in N$. By Lemma 5 and equation (28), we have $e a \in C$, and hence $[e a, x]=0$ for all $x \in R$, which implies

$$
\left[c^{m} a, x\right]=c^{m}[a, x]=0, \quad \forall x \in R .
$$

The argument used in Case 1 of Theorem 6 shows that

$$
c^{m}[a, x]=0 \quad \text { implies }[a, x]=0,
$$

and hence (see (29))

$$
[a, x]=0 \quad \forall x \in R, \forall a \in N .
$$

Thus, $R$ is a periodic ring with the property that $N \subseteq C$. By a well-known theorem of Herstein [4], it follows that $R$ is commutative, and the theorem is proved.

COROLLARY 7. Suppose that $R$ is a generalized periodic ring with identity 1 . Then, $R$ is commutative.

Corollary 7 follows at once by taking $c=1$ in Theorem 6 .

Since a generalized Boolean ring is also a generalized periodic ring, therefore we have the following corollary.

COROLLARY 8. A generalized Boolean ring with identity 1 is necessarily commutative.

Another corollary is the following result, proved by the authors in [1]. 
COROLLARY 9. Suppose that $R$ is a generalized periodic ring containing a central element which is not a zero divisor. Then $R$ is commutative.

This follows at once, since the hypotheses of this corollary imply the hypotheses of Theorem 6 .

THEOREM 10. Suppose $R$ is a generalized periodic ring. Suppose, further, that there exists a nonzero central element $c$ such that

$$
c a=0 \quad \text { implies } a=0, \forall a \in N \text {. }
$$

Then $R$ is commutative.

PROoF. In [1], the authors proved the following result:

If $R$ is a generalized periodic ring, then the nilpotents

$N$ form an ideal and $R / N$ is commutative.

Let $x, y \in R$. By (33), for all $\bar{x}, \bar{y}$ in $R / N, \bar{x} \bar{y}=\bar{y} \bar{x}$, and hence $[x, y] \in N$. Taking $a=[x, y] \in N$ in (32), we see that (32) yields

$$
c[x, y]=0 \text { implies }[x, y]=0, \forall x, y \in R .
$$

The theorem now follows at once from Theorem 6.

THEOREM 11. A generalized Boolean ring $R$ with central idempotents is necessarily nil $(R=N)$ or commutative $(R=C)$.

Proof. Since $R$ is also a generalized periodic ring, therefore by Theorem 3, $R$ is commutative or periodic. If $R$ is commutative, there is nothing to prove. So we may assume that $R$ is periodic. We now distinguish two cases.

CASE $1(C \subseteq N)$. Recall that, by hypothesis, the set $E$ of idempotents is central, and hence $E \subseteq C \subseteq N$ (in the present case). Thus, $E \subseteq N$, and hence $E=\{0\}$. Therefore,

$$
\text { zero is the only idempotent of } R \text {. }
$$

Let $x \in R$. Since $R$ is periodic, therefore $x^{k}$ is idempotent for some positive integer $k$, and hence by (35), $x^{k}=0$, which proves that $R$ is nil.

CASE $2(C \nsubseteq N)$. Then, for some $c \in R$, we have

$$
c \in C, \quad c \notin N .
$$

Again, since $R$ is periodic, $c^{m}$ is idempotent for some positive integer $m$. Moreover, $c^{m} \neq 0$ (since $c \notin N$ ). The net result is (see (36))

$$
e=c^{m} \text { is a nonzero central idempotent of } R \text {. }
$$

Now, suppose $a \in N$. Since $0 \neq e \in C$ and $a \in N$, therefore $e+a \notin N$. Suppose, for the moment, that $a \notin C$. Then $e+a \notin C$ (since $e \in C$ ), and hence $e+a \notin(N \cup C)$. Therefore, by Definition 2,

$$
(e+a)-(e+a)^{n} \in(N \cap C), \quad \text { for some even integer } n \geq 2 .
$$


Since $R$ is also a generalized periodic ring, therefore by Lemma 5 (see (37))

$$
e a^{i} \in C, \quad \forall i \in\{1, \ldots, n-1\},\left(0 \neq e=e^{2}, e \in C, a \in N\right) .
$$

Combining (38) and (39), we see that

$$
a-a^{n} \in C, \quad \forall a \in N \backslash C .
$$

Since (40) is trivially satisfied for $a \in(N \cap C)$, therefore

$$
a-a^{n} \in C, \quad \forall a \in N, n \geq 2 .
$$

We claim that

$$
N \subseteq C
$$

The proof is by contradiction. Suppose (42) is false. Then, for some $a \in R$, we have

$$
a \in N, \quad a \notin C .
$$

Since $a \in N$, there exists a positive integer $\sigma_{0}$ such that

$$
a^{\sigma} \in C, \quad \forall \sigma \geq \sigma_{0}, \sigma_{0} \text { minimal. }
$$

Moreover, since $a \notin C$ (see (43)), therefore $\sigma_{0}>1$. Now, applying (41) to the nilpotent element $a^{\sigma_{0}-1}$, we see that

$$
a^{\sigma_{0}-1}-\left(a^{\sigma_{0}-1}\right)^{n} \in C, \quad \text { for some } n=n\left(a^{\sigma_{0}-1}\right) \geq 2 .
$$

Furthermore, since $\left(\sigma_{0}-1\right) n \geq\left(\sigma_{0}-1\right) 2 \geq \sigma_{0}$ (since $\left.\sigma_{0} \geq 2\right)$, (44) implies that

$$
\left(a^{\sigma_{0}-1}\right)^{n}=a^{\left(\sigma_{0}-1\right) n} \in C .
$$

Combining (45) and (46), we conclude that $a^{\sigma_{0}-1} \in C$, which contradicts the minimality of $\sigma_{0}$ in (44). This contradiction proves (42). Since $R$ is a periodic ring satisfying (42), therefore, by a well-known theorem of Herstein [4], $R$ is commutative. This completes the proof.

COROLLARY 12. A generalized Boolean ring with central idempotents and commuting nilpotents is commutative.

This corollary recovers a result proved by the authors in [1].

COROLLARY 13. If $R$ is a generalized Boolean ring, and if $R$ is 2-torsion-free, then $R$ is nil or commutative.

Proof. We claim that all idempotents of $R$ are central. Suppose not, and suppose $e$ is a noncentral idempotent in $R$. Then $-e \notin(N \cup C)$, and hence (see Definition 2)

$$
(-e)-(-e)^{n} \in C, \quad n \text { even. }
$$

Thus, $2 e \in C$, and hence [2e,x] $=0$ for all $x$ in $R$. Since $R$ is 2-torsion-free, $2[e, x]=0$ implies $[e, x]=0$, and thus $e \in C$, a contradiction. This contradiction proves that all idempotents of $R$ are central, and hence $R$ is nil or commutative, by Theorem 11 . 
THEOREM 14. Let $R$ be a generalized Boolean ring in which every finite subring is either commutative or nil. Then $R$ is either commutative or nil.

Proof. By contradiction. Thus, suppose $R$ is a generalized Boolean ring such that every finite subring of $R$ is either commutative or nil. Suppose, further, that $R$ is not commutative and not nil either. By Theorem 11, there must exist a noncentral idempotent element $e$ in $R$, and hence $e \notin(C \cup N)$. Thus (see Definition 2), since $-e \notin$ $(C \cup N)$,

$$
(-e)-(-e)^{n} \in(N \cap C), \quad n \text { even. }
$$

This implies that $2 e \in(N \cap C)$, and hence $(2 e)^{k}=2^{k} e=0$, for some $k \in \mathbb{Z}^{+}$. Since $e \notin C$, we must have the following:

$$
\text { Either } e x \text {-exe } \neq 0 \text { for some } x \in R \text {, or } x^{\prime} e-e x^{\prime} e \neq 0 \text { for some } x^{\prime} \in R \text {. }
$$

Suppose $u=e x-e x e \neq 0$. Then,

$$
e u=u \neq 0=u e=u^{2}, \quad(u=e x-e x e \neq 0) .
$$

Moreover,

$$
2 u=[2 e, e x]=0 \quad(\text { since } 2 e \in C) .
$$

Furthermore, the subring generated by $e$ and $u$ is

$$
\langle e, u\rangle=\{r e+s u \mid r, s \in \mathbb{Z}\} .
$$

Since $2^{k} e=0$ and $2 u=0$, the subring $\langle e, u\rangle$ is finite. Indeed,

$$
\langle e, u\rangle=\left\{r e+s u \mid 1 \leq r \leq 2^{k}, 1 \leq s \leq 2\right\} .
$$

On the other hand, if $x^{\prime} e-e x^{\prime} e \neq 0$ for some $x^{\prime} \in R$ (the only other possibility), then the subring, $\langle e, v\rangle$, generated by $e$ and $v=x^{\prime} e-e x^{\prime} e$ is (as is readily verified)

$$
\langle e, v\rangle=\left\{r e+s v \mid 1 \leq r \leq 2^{k}, 1 \leq s \leq 2\right\} .
$$

Again, $\langle e, v\rangle$ is a finite subring of $R$. Hence, in either case, we found a finite subring of $R$, which is neither commutative (since $e \notin C$ ), nor nil (since $e \notin N$ ), contradicting our hypothesis. This contradiction proves the theorem.

REMARK 15. A careful examination of the proof of Theorem 14 shows that we only need to assume that "every subring $S$, with $|S|=2^{m}$ for some positive integer $m$, is commutative or nil" in order for the ground generalized Boolean ring $R$ to be commutative or nil. Indeed, $|\langle e, u\rangle|=2^{k} \cdot 2=2^{k+1}$, since the representation of any $x$ in this subring in the form $x=r e+s u ; r, s \in \mathbb{Z}$, is unique. For, suppose $x=r e+s u$ and $x=r^{\prime} e+s^{\prime} u$. Then, $\left(r-r^{\prime}\right) e=\left(s^{\prime}-s\right) u$. Recall that $2 u=0$, and $u e=0$. Thus, if $s^{\prime}-s$ is even, then $\left(r-r^{\prime}\right) e=0$, and hence $r e=r^{\prime} e, s u=s^{\prime} u$. On the other hand, if $s^{\prime}-s$ is odd, then $\left(r-r^{\prime}\right) e=u$, and hence $\left(r-r^{\prime}\right) e e=u e=0$. Again, we obtain $r e=r^{\prime} e, s u=s^{\prime} u$.

We conclude with the following examples. 
EXAMPLE 16. Let

$$
R=\left\{\left(\begin{array}{ccc}
a & b & c \\
0 & a^{2} & 0 \\
0 & 0 & a
\end{array}\right): a, b, c \in \mathrm{GF}(4)\right\}
$$

It is readily verified that the idempotents of $R$ are central and

$$
x-x^{7}=0, \quad \forall x \in R \backslash(N \cup C),
$$

but $R$ is neither nil nor commutative. Hence, Theorem 11 is not true if we drop the hypothesis that " $n$ is even" in the definition of a generalized Boolean ring.

EXAMPLE 17. Let

$$
R=\left\{\left(\begin{array}{ccc}
0 & a & b \\
0 & 0 & c \\
0 & 0 & 0
\end{array}\right): a, b, c \in \mathrm{GF}(3)\right\}
$$

This example shows that we cannot drop the hypothesis that " $N$ is commutative" in Corollary 12. (Note that $R$ is not commutative.)

EXAMPLE 18. Let

$$
R=\left\{\left(\begin{array}{ll}
0 & 0 \\
0 & 0
\end{array}\right),\left(\begin{array}{ll}
1 & 0 \\
1 & 0
\end{array}\right),\left(\begin{array}{ll}
0 & 1 \\
0 & 1
\end{array}\right),\left(\begin{array}{ll}
1 & 1 \\
1 & 1
\end{array}\right): 0,1 \in \mathrm{GF}(2)\right\}
$$

This example shows that we cannot drop the hypothesis that "the idempotents are central" in Corollary 12. (Note that $R$ is not commutative.) This example also shows that we cannot drop the hypothesis that " $R$ is 2-torsion-free" in Corollary 13. Note that, in this ring $R, x-x^{2}=0$ for all $x \in R \backslash(N \cup C)$. Even more is true. This ring $R$ also shows that we cannot drop the hypothesis that " $1 \in R$ " in Corollary 7 , nor the hypothesis that " $1 \in R$ " in Corollary 8 .

Returning to the ring $R$ in Example 16, we see that this ring further shows that we cannot drop the hypothesis that " $m$ and $n$ are of opposite parity" in the definition of a generalized periodic ring in connection with Corollary 7 , or the hypothesis that " $n$ is even" in the definition of a generalized Boolean ring as far as Corollary 8 is concerned. (Recall that $x-x^{7}=0$ for all $x \in R \backslash(N \cup C)$.)

EXAMPLE 19. Let $S$ be any noncommutative ring such that $S^{3}=(0)$. (For example, we may take $S$ to be the ring of all $3 \times 3$ strictly upper triangular matrices over a field $F$.) Let $R=\mathrm{GF}(4) \oplus S$. It is readily verified that $x^{3}=x^{6}$ for all $x$ in $R$, and hence $R$ is indeed a generalized periodic ring. Moreover, the only idempotents of $R$ are $(0,0)$ and $(1,0)$, and thus the idempotents of $R$ are certainly central. Had $R$ been a generalized Boolean ring, then, by Theorem $11, R$ would have to be either nil or commutative, which is certainly false here (recall that $S$ is not commutative). This example shows that the set of generalized periodic rings is a wider class than that of generalized Boolean rings, and thus Theorem 11 does not hold for generalized periodic rings. 
ACKNOWLEDGEMENT. This work was supported by the Natural Sciences and Engineering Research Council of Canada, Grant No. 3961.

\section{REFERENCES}

[1] H. E. Bell and A. Yaqub, Generalized periodic rings, Int. J. Math. Math. Sci. 19 (1996), no. 1, 87-92. MR 96h:16036. Zbl 842.16019.

[2] M. Chacron, On a theorem of Herstein, Canad. J. Math. 21 (1969), 1348-1353. MR 41 \#6905. Zbl 213.04302.

[3] I. N. Herstein, A generalization of a theorem of Jacobson. III, Amer. J. Math. 75 (1953), 105-111. MR 14,613e. Zbl 050.02901.

[4] _ A note on rings with central nilpotent elements, Proc. Amer. Math. Soc. 5 (1954), 620. MR 16,5c. Zbl 055.26003.

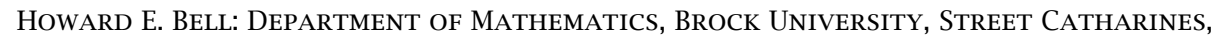
ONTARIO, CANADA L2S 3A1

E-mail address: hbe11@spartan.ac.brocku.ca

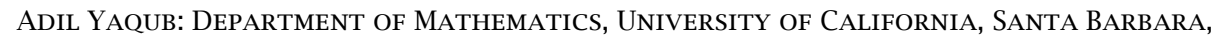
CA 93106, USA

E-mail address: yaqub@math . ucsb . edu 


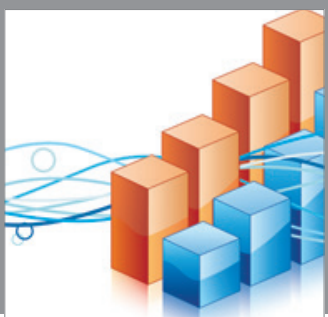

Advances in

Operations Research

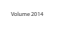

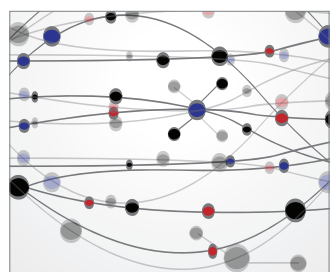

\section{The Scientific} World Journal
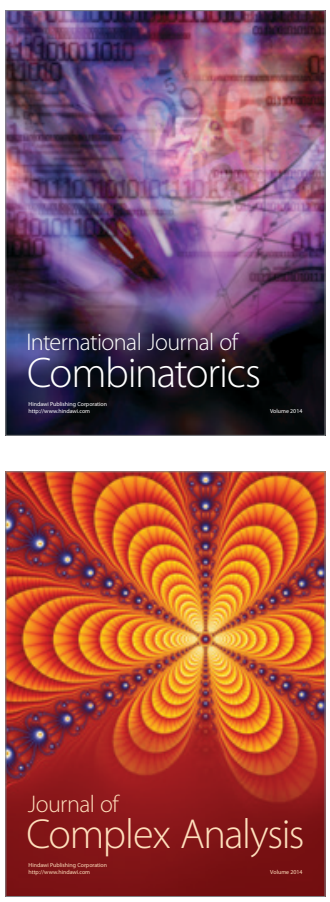

International Journal of

Mathematics and

Mathematical

Sciences
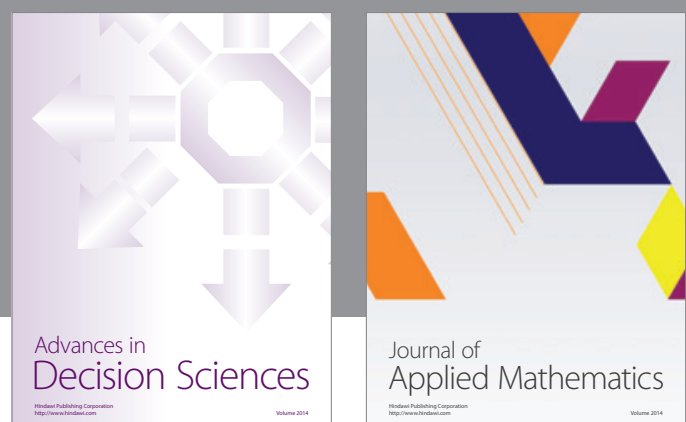

Journal of

Applied Mathematics
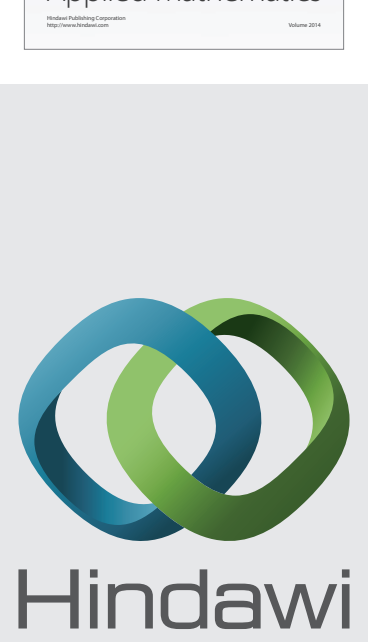

Submit your manuscripts at http://www.hindawi.com
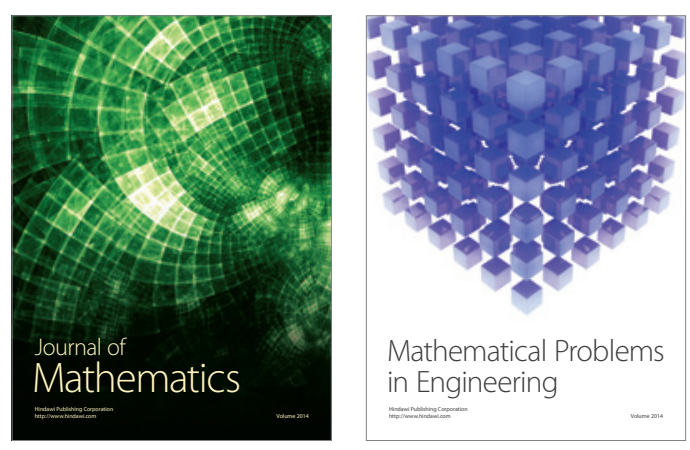

Mathematical Problems in Engineering
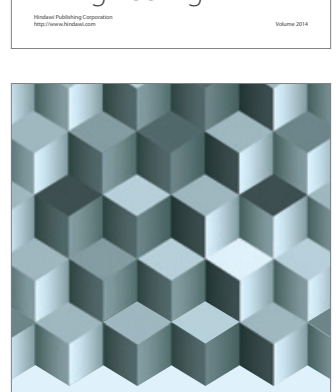

Journal of

Function Spaces
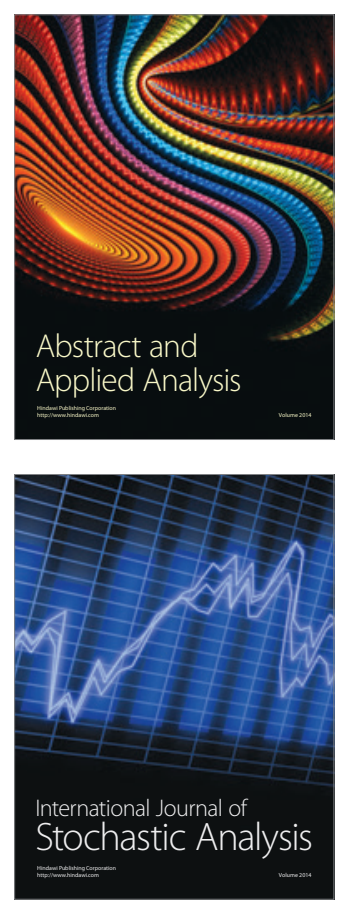

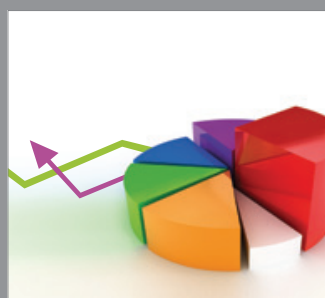

ournal of

Probability and Statistics

Promensencen
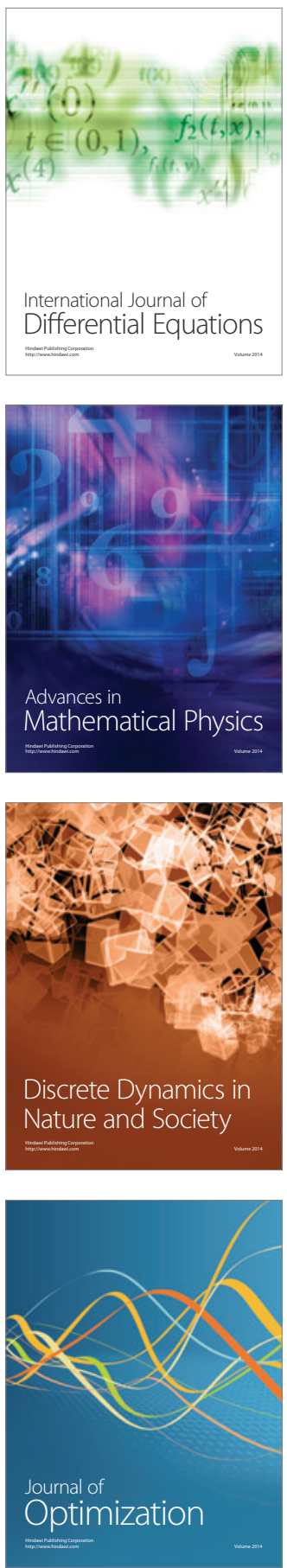